\title{
Effect of magnesium sulfate given for neuroprotection before pretermbitth: a randomized controlled trial (1)
}

\author{
Crowther CA, Hiller JE, Doyle LW, Haslam RR; Australasian Collaborative Trial of Mag- \\ nesium Sulphate (ACTOMgSO4) Collaborative Group. JAMA 2003;290:2669-76.
}

Análisis crítico: Paula Sperry $S^{1}$. Jorge Carvajal $C^{1}, P h D$. Claudio Vera $P-G^{1,2}, M S c$.

\footnotetext{
1 Unidad de Medicina Materno Fetal, División de Obstetricia y Ginecología, ${ }^{2}$ Unidad de Medicina Basada en Evidencia, Facultad de Medicina, Pontificia Universidad Católica de Chile.
}

\section{RESUMEN (1)}

Antecedentes: El sulfato de magnesio prenatal puede reducir el riesgo de parálisis cerebral o muerte en infantes prematuros severos. Objetivo: Determinar la efectividad del sulfato de magnesio, administrado para neuroprotección a mujeres en riesgo de parto prematuro antes de las 30 semanas, para prevenir mortalidad pediátrica y parálisis cerebral. Método: Estudio controlado randomizado en 16 centros terciarios en Australia y Nueva Zelanda, con estratificación por centro y embarazo múltiple. Un total de 1.062 mujeres con fetos menores a 30 semanas de gestación, para quienes el parto fue planeado o esperado dentro de 24 horas, fueron enroladas entre febrero 1996 a septiembre 2000, con seguimiento de los niños sobrevivientes a 2 años de edad corregida. Intervenciones: Mujeres fueron aleatoriamente asignadas a recibir una dosis de carga de $8 \mathrm{~mL}(4 \mathrm{~g}$ [16 mmol] de $0,5 \mathrm{~g} / \mathrm{mL})$ de solución de sulfato de magnesio o solución isotónica de cloruro de magnesio $(0,9 \%)$ por 20 minutos seguido de una infusión de mantención de $2 \mathrm{~mL} / \mathrm{h}$ por hasta 24 horas. Principales medidas de resultado: Tasas de mortalidad pediátrica total, parálisis cerebral y el resultado combinado de muerte o parálisis cerebral a la edad corregida de 2 años. $R e-$ sultados: Los datos fueron analizados para 1.047 (99\%) de los sobrevivientes a 2 años. Mortalidad pediátrica total ( $13,8 \%$ vs. $17,1 \%$; RR: 0,$83 ; 95 \%$ IC: $0,64-1,09)$, parálisis cerebral en sobrevivientes $(6,8 \%$ vs $8,2 \%$; RR: 0,$83 ; 95 \%$ IC: $0,54-1,27)$ y muerte o parálisis cerebral combinados $(19,8 \%$ vs 24,0\%; RR: 0,83; 95\% IC: 0,66-1,03) fueron menos frecuentes en infantes expuestos a sulfato de magnesio, pero ninguna de las diferencias fue estadísticamente significativa. Disfunción motora gruesa sustancial (3,4\% vs $6,6 \%$; RR: 0,51 ; $95 \%$ IC: 0,29 $0,91)$ y muerte o disfunción motora gruesa sustancial combinados ( $17,0 \%$ vs $22,7 \%$; RR: 0,75 ; $95 \%$ IC: $0,59-0,96)$ fueron significativamente reducidos en el grupo magnesio. Conclusiones: El sulfato de magnesio administrado a mujeres inmediatamente antes de un parto prematuro extremo puede mejorar importantes resultados pediátricos. No se observaron efectos adversos serios.

\section{ANÁLISIS DE LA INVESTIGACIÓN}

\section{A. Relevancia clínica de la investigación}

$¿$ En embarazadas en alto riesgo de parto prematuro temprano viable, la profilaxis antenatal con sulfato de magnesio comparado con placebo, es capaz de prevenir el desarrollo de parálisis cerebral en sus hijos?

Escenario clínico: Los recién nacidos prematuros presentan mayor riesgo de mortalidad, y entre aquellos que sobreviven, mayores tasas de parálisis cerebral, impedimentos neurosensoriales y discapacidad $(2,3)$. Los principales factores de riesgo para parálisis cerebral son la prematurez extrema y el muy bajo peso de nacimiento. El riesgo de compromiso neurológico es mayor a menor edad 
gestacional; hasta un tercio de todos los casos de parálisis cerebral se asocian a recién nacidos de muy bajo peso, los cuales incluyen a los prematuros extremos (4). A modo de ejemplo, en Chile el seguimiento neurológico a 2 años de recién nacidos de muy bajo peso entre los años 1994 y 1996 del Hospital Dr. Sótero del Río mostró que la evaluación neurológica era anormal en 32\% de los 181 sobrevivientes evaluados, con $24 \%$ de anormalidad neurosensorial severa y $6,6 \%$ de parálisis cerebral (5). La evidencia observacional que asoció a niños expuestos a sulfato de magnesio antes de su nacimiento prematuro, con un menor riesgo de parálisis cerebral (6) fue el argumento inicial para evaluar esta intervención con estudios controlados randomizados. Hasta el momento de la publicación analizada no existían estudios clínicos randomizados grandes que avalaran esta hipótesis (7).

\section{B. El estudio (1)}

Diseño: Estudio clínico randomizado, prospectivo, multicéntrico, controlado con placebo y ciego para los pacientes, clínicos y evaluadores de los eventos de interés. Pacientes: Embarazos únicos a cuádruples, con una edad gestacional desde el límite de viabilidad (determinado por cada centro) hasta las 30 semanas de gestación, en alto riesgo de parto prematuro espontáneo o con indicación de interrupción en las próximas 24 horas. No fueron elegibles aquellas mujeres que se encontraban ya en la segunda etapa del trabajo de parto, hubiesen recibido previamente durante este embarazo sulfato de magnesio o tuvieran alguna contraindicación para recibirlo. Intervención: Sulfato de magnesio o placebo idéntico con carga de $4 \mathrm{~g}$ seguidos de mantención de $1 \mathrm{~g} / \mathrm{h}$. Si no ocurría el parto, la mantención se suspendía a las 24 horas y se reiniciaba con dosis de carga si la amenaza de parto reaparecía en más de 6 horas luego de haberse suspendido. Eventos de interés: Los eventos de interés primarios fueron mortalidad pediátrica, parálisis cerebral, y el resultado compuesto de ambos a los 2 años de edad corregida por prematurez. Para evaluar los eventos de interés secundarios se realizó una ecografía transcraneal dentro de la primera semana de vida, otra a las 4 semanas, y luego, a los dos años una evaluación pediátrica y psicológica con la aplicación de índices de desarrollo psicomotor y mental. Como eventos de interés secundarios se consideró la presencia de hemorragia intraventricular grado III o IV, leucomalasia periventricular, deshabilidad neurosensorial, y en la madre se consignó los efectos cardiovasculares, respiratorios y la asociación con metrorragia posparto. Otros eventos evaluados fueron otros efectos adversos asociados a la infusión, resultados del embarazo y otros resultados neonatales. Resultados: Un total de 1.062 embarazadas fueron aleatorizadas y el seguimiento se logró para el $99 \%$ de los niños. La tasa de los resultados primarios no resulto significativamente diferente en el grupo de sul-ffato de magnesio comparado con placebo, con mortalidad $13,8 \%$ y $17,1 \%$ respectivamente (RR: 0,83; IC 95\%: 0,64 a 1,09); parálisis cerebral $6,8 \%$ y $8,2 \%$ respectivamente (RR: 0,83; IC 95\%: 0,54 a 1,27); y resultado compuesto $19,8 \%$ y $24,0 \%$ respectivamente (RR: 0,83; 95\% IC: 0,66 a 1,03). En el análisis de los resultados secundarios, se evidenció una disminución significativa en la tasa de disfunción motora gruesa sustancial en los sobrevivientes a dos años y de la tasa compuesta de mortalidad y disfunción motora gruesa sustancial a dos años; con tasas de $3,4 \%$ y $6,6 \%$ respectivamente (RR: 0,51 ; IC $95 \%$ : 0,29 a 0,91 ); y para el evento compuesto $17 \%$ y 22,7\% respectivamente (RR: 0,75; IC 95\%: 0,59 a 0,96$)$. No se presentaron eventos adversos que comprometieran la vida materna ni fetal.

\section{Análisis Crítico}

Validez interna: Estudio clínico randomizado, con aleatorización generada por computador con bloques variables de 4, 6 y 8 ; estratificado por centro clínico y gemelaridad. Se resguardó el ocultamiento de la secuencia por medio de contenedores secuenciales codificados que ocultaban bolsas de infusión de igual apariencia. Pacientes, clínicos y evaluadores del evento de interés fueron ciegos, con un seguimiento de $99 \%$ del total de población randomizada, similar en ambos grupos, análisis por de intención de tratar, incluyendo aquellos fetos vivos al momento de la randomización, independiente si nacía prematuro o no. Se realizó control de variables confundentes, ajustando por raza, hospital, beneficiaria pública o no, rotura prematura de membranas y metrorragia como causa de parto prematuro, ya que en el caso de la evaluación de mortalidad su desbalance demostró estar asociado con ese evento de interés. Métodos estadísticos: El cálculo de tamaño muestral se realizó pensando en detectar un $50 \%$ de disminución del riesgo de parálisis cerebral a los dos años corregidos, en los sobrevivientes, presupuestando que disminuiría de 10 a $5 \%$, con $80 \%$ de probabilidad con nivel alfa 0,05 . Se realizó una estimación conservadora de disminución esperada, dado el $86 \%$ de disminución de parálisis cerebral reportado por estudios observacionales previos. Finalmente se ajustó la muestra requerida teniendo en cuenta un $20 \%$ de mortalidad 
y los embarazos múltiples. El tamaño se alcanzó completamente y el estudio no se terminó prematuramente. Se describe cuales análisis estaban preespecificados y cuales se realizaron posteriormente. Comentarios: Estudio randomizado controlado de alta calidad metodológica y baja probabilidad de sesgo, donde no se observó reducción significativa de los eventos de interés primarios. Sin embargo, las tasas de parálisis cerebral y mortalidad previstas, fueron menores que las estimadas, lo cual afectó el poder del estudio para detectar diferencias que pudiesen ser clínicamente significativas. Así, el beneficio observado fue menor al esperado según los estudios observacionales previos. Debe tenerse en cuenta que la capacidad diagnóstica de PC a los dos años no alcanza un $100 \%$, lo que también pudo afectar los resultados. Dentro de los resultados secundarios se evidenció una disminución significativa en la tasa de disfunción motora gruesa sustancial a dos años y en la tasa compuesta de mortalidad y disfunción motora gruesa sustancial, ambos resultados importantes para los pacientes. Adicionalmente no se detectó efectos deletéreos para la madre ni el feto. El potencial efecto del sulfato de magnesio administrado a la madre para neuroprotección en el feto en riesgo de parto prematuro ha sido reevaluado en al menos 4 revisiones sistemáticas, sin encontrar beneficio en la reducción de mortalidad global fetal y neonatal-infantil. La actualización reciente de la revisión sistemática Cochrane incluye 5 estudios controlados randomizados (8) dos de ellos calificados de alta calidad metodológica y bajo riesgo de sesgo: el estudio analizado en esta oportunidad y el estudio de Rouse y cols (9) cuya evaluación de riesgo de sesgo está basada en los datos no publicados del protocolo. Para el evento de parálisis cerebral el meta-análisis reporta una reducción significativa (RR: 0,68; IC 95\%: 0,54 a 0,87$)$. En base a los resultados de los estudios el Colegio Americano de Obstetras y Ginecólogos junto con la Sociedad de Medicina Materno-Fetal han reconocido el potencial beneficio neuroprotector de sulfato de magnesio antenatal para disminuir el riesgo de parálisis cerebral y también la dificultad de recomendar algún esquema de tratamiento y seguimiento (10). Recientemente Huusom y cols (11) han realizado observaciones relevantes sobre el potencial riesgo de error al azar del meta-análisis de los estudios incluidos en la revisión Cochrane basado en un análisis secuencial de estudios, resaltando la necesidad de nuevos estudios que per- mitan reducir el error al azar y así obtener la evidencia necesaria para rechazar o aprobar su uso en forma sólida. Conclusión: Estudio bien diseñado, sin fuentes evidentes de sesgo, pero no suficiente para recomendar el uso de sulfato de magnesio como neuroprotector.

\section{BIBLIOGRAFÍA}

1. Crowther CA, Hiller JE, Doyle LW, Haslam RR; Australasian Collaborative Trial of Magnesium Sulphate (ACTOMgSO4) Collaborative Group. Effect of magnesium sulfate given for neuroprotection before pretermbirth: a randomized controlled trial. JAMA 2003;290:2669-76.

2. Lorenz JM, Wooliever DE, Jetton JR, Paneth N. A quantitative review of mortality and developmental disability in extremely premature newborns. Arch $\mathrm{Pe}$ diatr Adolesc Med 1998;152:425-35.

3. Drummond PM, Colver AF. Analysis by gestational age of cerebral palsy in singleton births in northeast England 1970-94. Paediatr Perinat Epidemiol 2002;16:172-80.

4. Hack M, Costello DW. Trends in the rates of cerebral palsy associated with neonatal intensive care of pre 7 term children. Clin Obstet Gynecol 2008;51:763-74.

5. Alegría A, Pittaluga E, Mena $P$, Schlack L, Díaz $M$, Vergara M, Dapremont I, Jiménez E. Evolución neurosensorial en recién nacidos de muy bajo peso de nacimiento a los 2 años de edad. Rev Chil Pediatr 2002;73:348-56.

6. Nelson KB, Grether JK. Can magnesium sulfate re $\neg$ duce the risk of cerebral palsy in very low birthweight infants? Pediatrics 1995;95:263-9.

7. Doyle LW, Crowther CA, Middleton $P$, Marret $S$. Mag $\neg$ nesium sulphate for women at risk of preterm birth for neuroprotection of the fetus. Cochrane Database Syst Rev 2007;3:CD004661.

8. Doyle LW, Crowther CA, Middleton P, Marret S, Rouse D. Magnesium sulphate for women at risk of preterm birth for neuroprotection of the fetus. Cochrane Database Syst Rev 2009;1:CD004661

9. Rouse DJ, Hirtz DG, Thom E, Varner MW, Spong $\mathrm{CY}$, Mercer BM, et al. A randomized, controlled trial of magnesium sulfate for the prevention of cerebral palsy. N Engl J Med 2008;359:895-905.

10. American College of Obstetricians and Gynecologists Committee on Obstetric Practice; Society for Maternal-Fetal Medicine. Committee Opinion No. 455: Magnesium sulfate before anticipated preterm birth for neuroprotection. Obstet Gynecol 2010;115:669-71.

11. Huusom LD, Secher NJ, Pryds O, Whitfield K, Gluud $\mathrm{C}$, Brok J. Antenatal magnesium sulphate may prevent cerebral palsy in preterm infants--but are we convinced? Evaluation of an apparently conclusive meta-analysis with trial sequential analysis. BJOG 2011;118:1-5. 